\title{
Mask Ventilation Grip: A Life-Saving Innovation
}

\author{
Jacquelin Peck, MD, ${ }^{1}$ Omar Viswanath, MD, ${ }^{2}$ Gerald Rosen, MD ${ }^{3}$ \\ ${ }^{1}$ Department of Anesthesiology, Johns Hopkins All Children's Hospital, St. Petersburg, FL ${ }^{2}$ Department of Anesthesiology, Critical Care, and \\ Pain Medicine, Beth Israel Deaconess Medical Center, Boston, MA ${ }^{3}$ Department of Anesthesiology, Mt. Sinai Hospital, Miami Beach, FL
}

\section{TO THE EDITOR}

Efficient and successful bag-mask ventilation (BMV) is an essential skill that can save a patient's life when seconds count. Ventilation is used by practitioners in a multitude of areas, including perioperative areas (specifically the operating room [OR] during induction of general anesthesia and extubation), intensive care units, and emergency departments, as well as by rapid response and code blue teams on the wards and by emergency medical services (EMS) first responders for out-of-hospital resuscitation efforts. BMV is a skill that requires extensive training and repetitionbased practice to perform properly. ${ }^{1,2}$

Improper BMV may lead to hypoventilation, hypoxemia, acidosis, hypoxic brain injury, cardiac arrest, and even death. Improper BMV manifests in inadequate ventilation (delivery of appropriate-sized breaths known as tidal volume), and a faulty mask seal could lead to hypoxemia and death. ${ }^{1}$

A 2011 study demonstrated that medical professionals without up-to-date BMV training perform BMV successfully only $16.6 \%$ of the time. ${ }^{3}$ Because of its difficulty, BMV is also frequently performed unsuccessfully by laypersons or abandoned in cardiopulmonary resuscitation (CPR) situations. ${ }^{4}$ Even trained medical professionals frequently experience difficulty. Success further deteriorates in simulated OR situations without the use of technology such as capnography and tidal volume monitoring to guide performance. ${ }^{3,5,6}$ Similarly, trained EMS providers frequently experience difficulty maintaining a mask-face seal and deliver adequate tidal volumes without air leak only $26.7 \%$ of the time. ${ }^{1}$

Difficulty maintaining an adequate mask-face seal has a strong association with physical characteristics of both the provider and the patient. ${ }^{7}$ Physical traits that create difficulty include the provider's sex, height, weight, hand size, and grip strength. ${ }^{8}$ Patient characteristics that create higher rates of BMV difficulty include facial hair, lack of teeth, large neck circumference, obesity, sleep apnea, excessive pharyngeal tissue, inability to flex or extend the head, mandibular immobility, and old age. ${ }^{7,9,10}$ Using a two-handed mask-face sealing technique has demonstrated the greatest success in delivering consistent, adequate tidal volumes during BMV in difficult patients as well as in the emergency department and out-of-hospital situations. ${ }^{11,12}$

Documented complications of BMV are gastric inflation and aspiration of stomach contents. ${ }^{7}$ Gastric inflation may be reduced by using a smaller bag; however, standard ventilation kits contain large bags because inexperienced users struggle to form an effective seal and therefore require a large bag to deliver the required tidal volume after air leak. ${ }^{5}$ Assessment of proper BMV in the controlled environment of patient simulation has proven difficult because of the facial structure of mannequins coupled with the inability of simulation technology to measure exhaled tidal volumes. The use of an adjunct to improve BMV seal has the potential to allow for greater standardization of tidal volume delivery and improved patient safety.

\section{INNOVATION}

The Mask Ventilation Grip is a disposable adjunct that is designed to fit all standard ventilation masks. The device is inexpensive and universally adaptable for emergency, CPR, and anesthesiology services. Its purpose is to help healthcare practitioners adequately mask-ventilate patients, independent of skill level. The Mask Ventilation Grip fits over standard masks (Figure) and reduces the BMV learning curve by guiding correct hand positioning with the wings of the device in the palms or thenar eminences and the fingers gripping the mandibular angles. This position ensures a strong mask-face seal while allowing for anterior mandibular advancement to maintain a patent airway.

The device was designed by an anesthesiologist (Gerald Rosen, MD, founding program director of the Mount Sinai Medical Center Anesthesiology Residency Program) after responding to many cardiopulmonary arrests and prearrest situations in the hospital setting and witnessing healthcare practitioners across all levels-respiratory therapists, nursing staff, medical students, residents, fellows, and even attending physicians - who were unable to create a sufficient mask-face seal and adequately mask-ventilate patients prior to intubation.

The goal of the Mask Ventilation Grip is to prevent or limit episodes of hypoventilation by improving the ease and success rates of BMV. It is an add-on device that ensures proper technique and reduces or removes the learning curve for lesser-trained individuals. It also assists anesthesiologists and other advanced airway specialists when confronted with patients who have anatomical challenges, as well as practitioners with a small hand size relative to a patient's facial anatomy. Use of the device is also intended to prevent hand fatigue with prolonged BMV.

\section{STAGES OF DEVELOPMENT}

The Mask Ventilation Grip started as a concept and then progressed through several 2-dimensional markups until the inventor settled on the current form that was converted to a 3-dimensional form with a 3-dimensional printer. The 


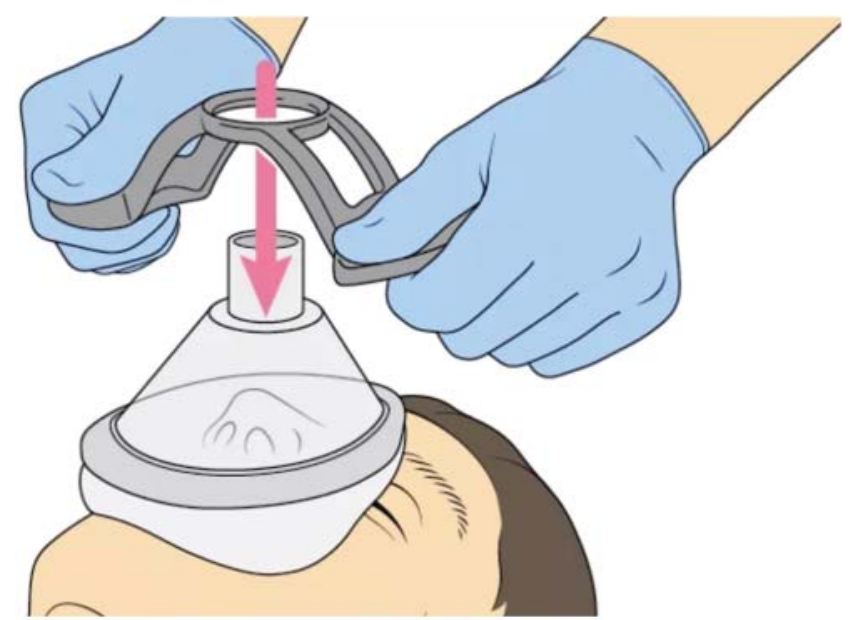

Figure. The Mask Ventilation Grip is placed over any standard valve mask.

Mask Ventilation Grip is now patent approved for design and utility. The Mask Ventilation Grip is currently listed and registered with the US Food and Drug Administration as a Class I exempt device.

\section{NEXT STEPS}

Two institutional review board-approved simulation studies are currently underway to evaluate the Mask Ventilation Grip at Mount Sinai Medical Center of Florida and at Florida International University, School of Nurse Anesthesia, in Miami, FL. Human studies and a soft rollout of the product are in the conceptual phase of planning. Targeted markets will include hospital and ambulatory procedural suites, as well as emergency departments, EMSs, and code carts. For more information, visit www.otegmedical.com.

\section{ACKNOWLEDGMENTS}

Dr Gerald Rosen is the founder of Oteg Medical LLC and is the inventor and patent holder of the Mask Ventilation Grip.

\section{REFERENCES}

1. Lee HY, Jeung KW, Lee BK, et al. The performances of standard and ResMed masks during bag-valve-mask ventilation. Prehosp Emerg Care. 2013 Apr-Jun;17(2):235-240. doi: 10.3109/10903127.2012.729126.
2. Khoury A, De Luca A, Sall FS, Pazart L, Capellier G. Performance of manual ventilation: how to define its efficiency in bench studies? A review of the literature. Anaesthesia. 2015 Aug;70 (8):985-992. doi: 10.1111/anae.13097.

3. Soleimanpour H, Gholipouri C, Panahi JR, et al. Role of anesthesiology curriculum in improving bag-mask ventilation and intubation success rates of emergency medicine residents: a prospective descriptive study. BMC Emerg Med. 2011 Jun 16;11:8. doi: 10.1186/1471-227X-11-8.

4. Fuchs $P$, Obermeier J, Kamysek S, et al. Safety and applicability of a pre-stage public access ventilator for trained laypersons: a proof of principle study. BMC Emerg Med. 2017 Dec 4;17(1):37. doi: 10.1186/s12873-017-0150-5.

5. Zobrist B, Casmaer M, April MD. Single rescuer ventilation using a bag-valve mask with internal handle: a randomized crossover trial. Am J Emerg Med. 2016 Oct;34(10):1991-1996. doi: 10.1016/j.ajem.2016.07.030.

6. Mumma JM, Durso FT, Dyes M, Dela Cruz R, Fox VP, Hoey M. Bag valve mask ventilation as a perceptual-cognitive skill. Hum Factors. 2018 Mar;60(2):212-221. doi: 10.1177/ 0018720817744729.

7. Cattano D, Katsiampoura A, Corso RM, Killoran PV, Cai C, Hagberg CA. Predictive factors for difficult mask ventilation in the obese surgical population. F1000Res. 2014 Oct 9;3:239. doi: 10.12688/f1000research.5471.1.

8. Na JU, Han SK, Choi P, Cho JH, Shin DH. Influence of face mask design on bag-valve-mask ventilation performance: a randomized simulation study. Acta Anesthesiol Scand. 2013 Oct;57(9):1186-1192. doi: 10.1111/aas.12169.

9. Bucher JT, Cooper JS. Bag mask ventilation. StatPearls [Internet]. Treasure Island, FL: StatPearls Publishing; 2018 Jan-. https://www.ncbi.nlm.nih.gov/books/NBK441924/. Updated June 24, 2017. Accessed March 14, 2018.

10. Cattano D, Killoran PV, Cai C, Katsiampoura AD, Corso RM, Hagberg CA. Difficult mask ventilation in general surgical population: observation of risk factors and predictors. F1000Res. 2014 Aug 27;3:204. doi: 10.12688/ f1000research.5131.1.

11. Otten D, Liao MM, Wolken $R$, et al. Comparison of bag-valvemask hand-sealing techniques in a simulated model. Ann Emerg Med. 2014 Jan;63(1):6-12.e3. doi: 10.1016/j. annemergmed.2013.07.014.

12. Joffe AM, Hetzel S, Liew EC. A two-handed jaw-thrust technique is superior to the one-handed "EC-clamp" technique for mask ventilation in the apneic unconscious person. Anesthesiology. 2010 Oct;113(4):873-879. doi: 10.1097/ ALN.0b013e3181ec6414. 